\title{
HSTOF ENA observations and energetic ion distributions in the heliosheath ${ }^{\star}$
}

\author{
A. Czechowski ${ }^{1}$, M. Hilchenbach ${ }^{2}$, and K. C. Hsieh ${ }^{3}$ \\ ${ }^{1}$ Space Research Center, Polish Academy of Sciences, Bartycka 18A, 00716 Warsaw, Poland \\ e-mail: ace@cbk.waw.pl \\ 2 Max-Planck-Institut für Sonnensystemforschung, Max-Planck-Strasse 2, 37191 Katlenburg-Lindau, Germany \\ e-mail: hilchenbach@mps.mpg.de \\ 3 Physics Department, University of Arizona, Tucson, AZ 85721, USA \\ e-mail: hsieh@physics.arizona.edu
}

Received 3 December 2011 / Accepted 9 March 2012

\section{ABSTRACT}

\begin{abstract}
Context. Launched in 1996, HSTOF on board SOHO was the first instrument to detect the energetic neutral atoms (ENA) from the heliosheath. After mid 2003, the field of view of HSTOF was restricted to the flank sectors of the heliosheath, in which region the energetic ion distributions are still unknown. Interpretation of these data requires understanding of the energetic ion transport in the inner heliosheath.

Aims. We update the HSTOF ENA hydrogen and helium spectra by adding the results from the recent (2006-2010) measurements. We calculate the energetic ion distributions in a numerical model of the heliosheath and use them as a basis for interpreting the HSTOF ENA observations, in particular those of the flank sectors.

Methods. The hydrogen and helium ENA spectra were derived from the HSTOF observations of two $90^{\circ}$-wide ecliptic longitude sectors around the crosswind directions. The energetic ion distributions in the heliosheath were calculated in a simple model of the heliosphere, assuming that the main acceleration occurs at the termination shock. The effects of different processes (charge-exchange loss, adiabatic acceleration, parallel diffusion and escape across the boundary) were determined with different assumptions about the boundary conditions and the transport parameters. The resulting ion distributions were used to calculate the ENA fluxes, which can be compared with the observations by HSTOF and other instruments.

Results. The energetic ion density in the flank sectors of the heliosphere is lower than in the forward (upwind) sector. As a result, the contribution of the flanks of the heliosheath to the production of ENA is not directly proportional to the thickness of the heliosheath, but instead is comparable to the contribution of the forward sector, which agrees with the HSTOF data. The HSTOF ENA flux intensity is nevertheless significantly lower than the model calculations. Near the heliopause there appears a region of low energetic ion density caused by the charge-exchange and the escape losses.
\end{abstract}

Key words. acceleration of particles - solar wind - interplanetary medium

\section{Introduction}

Because of the low energetic neutral atoms (ENA) flux in the HSTOF (High energy Suprathermal Time Of Flight sensor) energy range $(58-88 \mathrm{keV}$ for $\mathrm{H}, 28-58 \mathrm{keV} / \mathrm{n}$ for $\mathrm{He})$, the ENA observations by HSTOF are possible only during quiet times, when the ion flux intensity is low (Hilchenbach et al. 1998, 2001). The quiet periods occur frequently only near solar minimum. In consequence, most of the ENA data obtained by HSTOF came from the first two years of operation $(1996,1997)$ and from the recent low-activity period (2006-2010). The reorientation of SOHO starting from mid 2003 made it impossible to observe the ENA coming from the forward part of the inner heliosheath (near the inflow direction of the interstellar medium: the upwind direction) or from the opposite sector (the heliotail). Recent observations are therefore restricted to the "flanks" of the heliosphere, the regions which, when viewed from the inner solar system, are situated near the directions perpendicular to the interstellar medium inflow (the crosswind directions).

* We dedicate this work to the memory of our longstanding coworker and dear colleague Karin Bamert.
The heliospheric ENA (Hsieh et al. 1992; Fahr et al. 2007) are produced predominantly by charge-exchange between the energetic ions and the neutral $\mathrm{H}$ atoms that enter the heliosphere from the interstellar medium. The ENA flux is therefore determined by the parent ion flux and the neutral $\mathrm{H}$ column density along the line of sight. Neutral He contribution to charge exchange is also non-negligible in the HSTOF energy range and becomes important in the heliotail sector.

After the termination shock crossing by Voyager 1 in the year 2004 (Stone et al. 2005; Decker et al. 2005) and by Voyager 2 in 2007 (Stone et al. 2008) the ion flux in the heliosheath is continuously being measured in situ. However, the Voyager measurements are restricted to the vicinity of their trajectories, which pass through the forward (upwind) part of the heliosheath.

Assuming that the energetic ion flux measured by the Voyager spacecrafts can be taken as an approximation to the average flux in the forward sector of the heliosheath, the Voyager ion data can be used to estimate the production rate of the ENA in this sector. Comparing these data with the ENA flux observed by HSTOF, one can estimate the column density of neutral $\mathrm{H}$ in the forward heliosheath. Czechowski et al. (2006a) used the early Voyager 1 data (Decker et al. 2005) and assumed that the 
proton flux is only $\sim 2 / 3$ of the $Z \geq 1$ Voyager flux. Hsieh et al. (2010) used more recent data and assumed the proton flux fraction to be 1 . As a result, the hydrogen column density obtained by Hsieh et al. (2010) is lower than in Czechowski et al. (2006a) and suggests that Voyager 1 may already be close to the heliopause (Hsieh et al. 2010).

We have attempted to extend this approach to the crosswind sectors (Czechowski et al. 2008), making the simplest possible (but not necessarily realistic) assumption that the average energetic ion flux in these sectors is the same as in the forward sector. We found that the neutral $\mathrm{H}$ column density in the crosswind direction (and consequently the thickness of the heliosheath) following from this assumption is comparable to, or lower than, in the forward sector. This result is in conflict with the generally accepted ideas about the structure of the heliosphere. In particular, most numerical models imply that the thickness of the heliosheath increases away from the forward direction (e.g. Baranov \& Malama 1993; Zank et al. 1996; Ratkiewicz et al. 1998; Zank 1999; Fahr et al. 2000; Pogorelov et al. 2008a,b) although the interstellar magnetic field may alter this in some cases (Izmodenov \& Alexashov 2006; Ratkiewicz \& Webb 2002).

The most probable conclusion is that in the HSTOF energy range, the average energetic ion flux in the crosswind sectors of the heliosheath is lower than in the forward sectors. To relate the ENA observations and the structure of the heliosheath requires understanding of the processes that shape the parent ion distributions.

In this work we present the results of HSTOF observations of the flank sectors of the heliosheath including also the most recent data. Because of the relatively low solar activity, the recent data (corresponding to the years 2006-2010) add considerably to the number of counts and so reduce the statistical errors. The helium spectra are obtained using the recent re-evaluation of ambient $\mathrm{He}^{+}$ion background.

We also present our calculations of the energetic $\mathrm{H}^{+}$and $\mathrm{He}^{+}$ ion distributions in the inner heliosheath. For this purpose we use the model of the heliosphere based on a gas-dynamical numerical solution (Fahr et al. 2000), which we have extended to include the heliospheric magnetic field and the interstellar neutral He background. The model is simple and offers only a rough approximation to the heliosheath structure and the ion distributions. However, the results provide useful order-of-magnitude estimations and offer suggestions for future, more complete studies.

In the framework of this model we estimate the effect and relative importance of charge-exchange neutralization loss, adiabatic acceleration, parallel diffusion and escape across the heliopause for the energetic ion distributions in the heliosheath. We find that the spatial distribution of the ions in the HSTOF energy range in the crosswind sectors of the heliosheath differs significantly from the forward sector.

We calculate the ENA fluxes corresponding to the derived ion distributions and compare them to HSTOF observations. We find that, despite significantly higher thickness of the heliosheath in the flank sectors, the calculated ENA fluxes from the flanks are comparable with those from the nose sector, similarly to the HSTOF data. The calculated fluxes are significantly higher in intensity than the corresponding HSTOF data, but the directional distribution is similar.

Although we concentrate on particles in the HSTOF energy range, we extend our calculations also to lower energies to compare to the IBEX (McComas et al. 2009; Schwadron et al. 2009, 2011) and INCA (Krimigis et al. 2009, 2010, 2011)
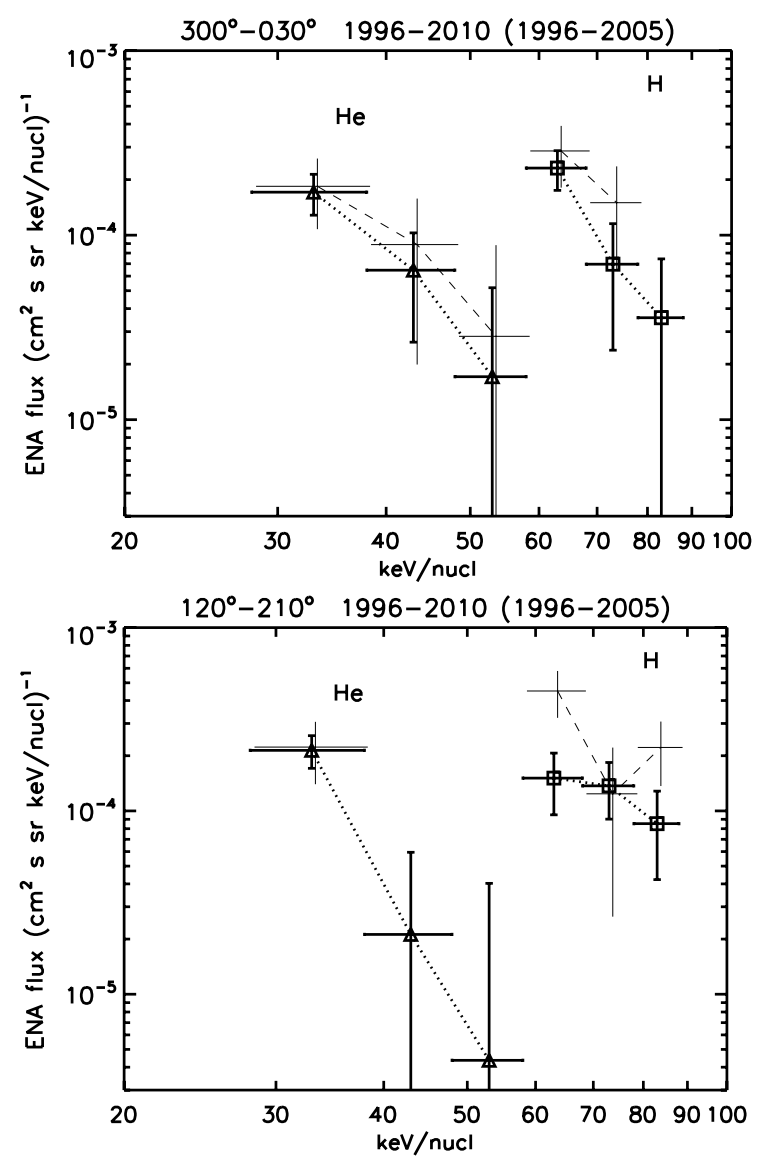

Fig. 1. HSTOF $\mathrm{H}$ and He ENA spectra for the flank sectors of ecliptic longitude $\left(300^{\circ}-30^{\circ}\right.$ and $\left.120^{\circ}-210^{\circ}\right)$ obtained over the period 1996-2010 (symbols, thick lines) and 1996-2005 (thin lines).

ENA data. We comment briefly on the relation between the lowenergy data and the observations by HSTOF.

\section{HSTOF ENA spectra from the flank sectors of the heliosphere}

HSTOF (Hovestadt et al. 1995) is part of the CELIAS experiment on board SOHO. The spacecraft is in orbit around the L1 point between the Earth and the Sun. The line of sight of HSTOF lies in the ecliptic plane, $37^{\circ}$ from the Earth-Sun direction. The field of view extends $\pm 17^{\circ}$ from the ecliptic and is $4^{\circ}$ wide in the ecliptic plane. Until the year 2003 the direction of the line of sight was fixed relative to the Sun and all ecliptic longitudes were scanned once a year.

From the year 2003 on it became necessary to change the spacecraft orientation (roll by $180^{\circ}$ ) at selected times. At each roll, the HSTOF line of sight direction is changed from $37^{\circ}$ west (east) to $37^{\circ}$ east (west) relative to the L1-Sun line. As a result, the forward (upwind) and the heliotail (downwind) sectors of the heliosphere became inaccessible to HSTOF observations: at the same time, parts of the flank (crosswind) sectors are scanned twice.

The ENA observations by HSTOF are restricted to the energy range $58-88 \mathrm{keV}$ for $\mathrm{H}$ and $28-58 \mathrm{keV} / \mathrm{n}$ for He.

Figure 1 shows the recently updated energy spectra of energetic neutral $\mathrm{H}$ and $\mathrm{He}$ averaged over two ecliptic longitude sectors: $120^{\circ}-210^{\circ}$ and $300^{\circ}-30^{\circ}$, corresponding to the flanks of the heliosphere. The data include all HSTOF ENA observations 


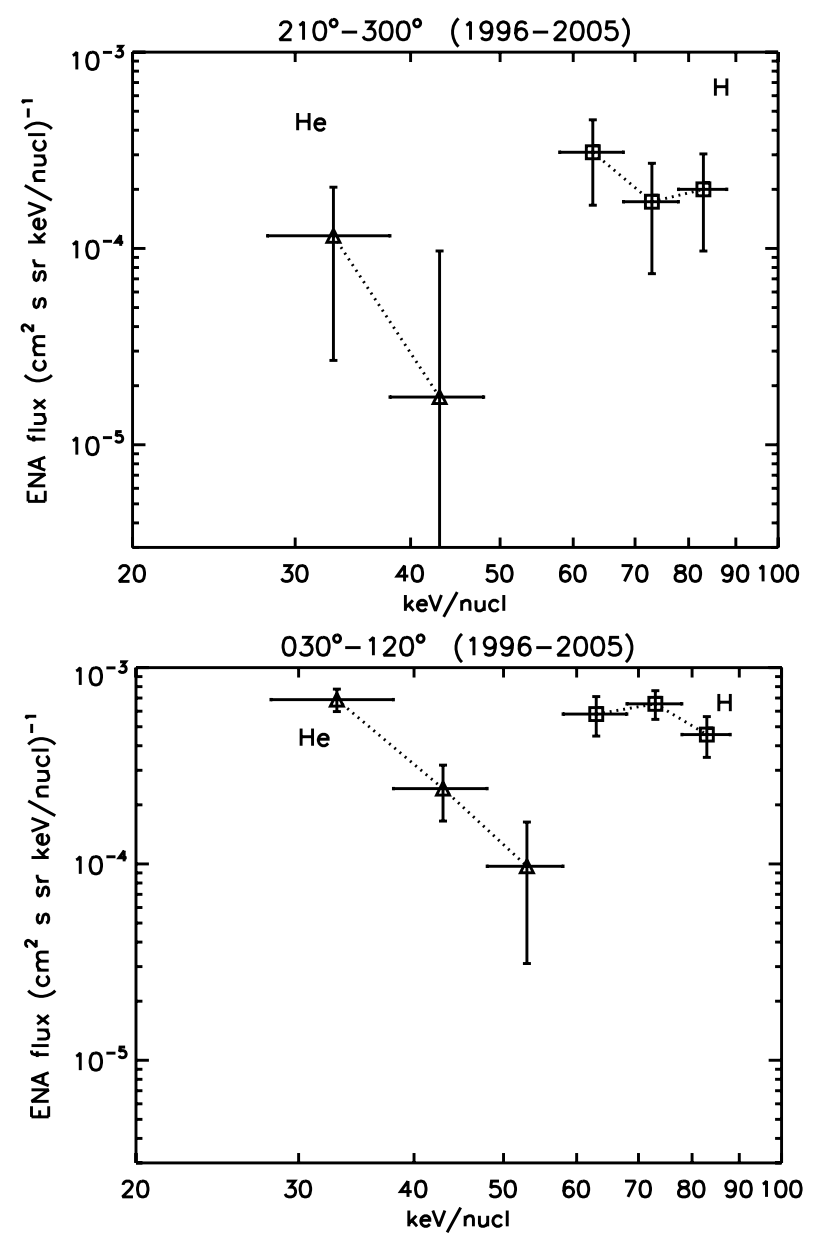

Fig. 2. HSTOF H and He ENA spectra for the ecliptic longitude sectors $210^{\circ}-300^{\circ}$ (forward sector) and $30^{\circ}-120^{\circ}$ (heliotail) obtained over the period 1996-2005.

of these sectors from the beginning of observations in the year 1996 until and including most of the year 2010. The data shown have the accidental background subtracted. The vertical error bars correspond to $1 \sigma$ statistical error. The horizontal bars show the $10 \mathrm{keV} / \mathrm{n}$ wide energy bins. Figure 1 also includes the corresponding ENA spectra for the period 1996-2005. The spectra for the forward (upwind) and the heliotail sectors (available only for the period 1996-2005) are shown in Fig. 2.

The helium ENA spectra shown in both figures were obtained using the recently re-evaluated background with higher contribution from the $\mathrm{He}^{+}$ambient ions (Hilchenbach et al. 2011). In consequence, the helium ENA spectra are different from those in Czechowski et al. (2008).

The hydrogen ENA flux from the flanks derived from the HSTOF data for the 1996-2010 time period is lower than the corresponding 1996-2005 flux because of low flux values registered during the 2006-2010 period. The energy-averaged $\mathrm{H}$ ENA flux for 1996-2010 is lower than the one for 1996-2005 by a factor $\sim 2$ (the $120^{\circ}-210^{\circ}$ flank sector) or $\sim 1.4$ (the $300^{\circ}-30^{\circ}$ sector). The largest difference occurs in the sector $120^{\circ}-210^{\circ}$ where it reaches $2.3 \sigma$ for the lowest energy (highest flux) data point. In the $300^{\circ}-30^{\circ}$ sector the difference between the 1996-2010 and 1996-2005 H ENA fluxes is within the $1 \sigma$ error bars.

In the 1996-2010 data the difference in the H ENA flux between the $300^{\circ}-30^{\circ}$ and $120^{\circ}-210^{\circ}$ sectors is reduced compared to the 1996-2005 time interval, so that the suggestion of the
Table 1. $\mathrm{H}^{+} / \mathrm{He}^{+}$ion flux ratio from HSTOF ENA data assuming a common $\mathrm{H}$ and $\mathrm{He}$ source region.

\begin{tabular}{lc}
\hline \hline Sector & $\mathrm{H}^{+} / \mathrm{He}^{+}$ \\
\hline $1996-2005$ & \\
$210^{\circ}-300^{\circ}$ & $28.6 \pm 24.1$ \\
$300^{\circ}-30^{\circ}$ & $12.8 \pm 6.1$ \\
$120^{\circ}-210^{\circ}$ & $16.0 \pm 7.0$ \\
$30^{\circ}-120^{\circ}$ & $10.6 \pm 1.8$ \\
\hline $1996-2010$ & \\
$300^{\circ}-30^{\circ}$ & $9.9 \pm 3.2$ \\
$120^{\circ}-210^{\circ}$ & $9.0 \pm 2.9$ \\
\hline
\end{tabular}

asymmetry between the two flanks made in Czechowski et al. (2008) is not confirmed.

We investigated the possibility that the downward shift in the H ENA flux is caused by a drop in the instrument sensitivity. However, a comparison with ACE-ULEIS over different time periods did not show a change large enough to account for the shift. The method used was the same as for in-flight calibration of HSTOF (Hilchenbach et al. 2001). We compared the correlation plots (ACE/ULEIS hourly averaged ion flux data plotted against the SOHO/HSTOF ion data) for two time intervals (1998-2005 and 2005-2010). Since the spectral hardness of the average solar energetic ion flux might have changed with time, we performed the comparison separately in two energy ranges ( 320 to 640 and 640 to $1280 \mathrm{keV}$ for $\mathrm{H}$ and 252 to 320 and 320 to $452 \mathrm{keV}$ for $\mathrm{He}$ ).

The helium ENA data for the periods 1996-2010 and 1996-2005 are quite similar. The lowest energy data points (the ones least affected by the ion contamination) for the 1996-2010 and 1996-2005 time intervals differ by only $5 \%$ (the $1 \sigma$ error is $\sim 40 \%$ for 1996-2005).

Table 1 shows the values of $\mathrm{H}^{+} / \mathrm{He}^{+}$ion flux ratio estimated from the HSTOF ENA data.

The $\mathrm{H}^{+} / \mathrm{He}^{+}$ratio is the ratio of fluxes in the source region at the same energy per nucleon. For the revised helium HSTOF ENA spectra it was first derived by Hilchenbach et al. (2011).

To estimate $\mathrm{H}^{+} / \mathrm{He}^{+}$from the ENA data requires some simplifying assumptions. The values in Table 1 were obtained as follows. We assumed that the $\mathrm{H}$ and He ENA observed by HSTOF are produced in the same source region predominantly by charge exchange between the parent ions $\left(\mathrm{H}^{+}\right.$and $\left.\mathrm{He}^{+}\right)$and the background neutral hydrogen. The ENA fluxes $J_{\mathrm{ENA}, i}(i=\mathrm{H}$ or $\mathrm{He})$ can be then approximately expressed in terms of the line-of-sight averaged parent ion fluxes $J_{\text {ion, } i}$ as $J_{\mathrm{ENA}, i}=J_{\text {ion, } i} \sigma_{\mathrm{cx}, i} N_{\mathrm{H}}$ where $\sigma_{\mathrm{cx}, i}$ is the charge exchange cross section between the ion and a neutral $\mathrm{H}$ atom and $N_{\mathrm{H}}$ the column density of neutral $\mathrm{H}$ in the source region. We also assumed that $J_{\text {ion, } i}$ for both $i=\mathrm{H}$ and He have the same energy dependence $E^{-1.6}$ in the HSTOF energy region. The ion fluxes and the $\mathrm{H}^{+} / \mathrm{He}^{+}$flux ratio were then obtained by fitting the $\mathrm{H}$ and He HSTOF ENA data.

The $\mathrm{H}^{+} / \mathrm{He}^{+}$ratio for the termination shock particles observed by Voyager 1 LECP estimated using the composite spectrum (Fig. 3 in Decker et al. 2005) is 25 (Czechowski et al. 2006a). Although the LECP energy range starts at $40 \mathrm{keV}$, this estimation requires extrapolation from higher energy $(\sim 1 \mathrm{MeV})$ since the lowest energy channels do not distinguish the species. The results from the Voyager 1 Cosmic Ray Subsystem give the $\mathrm{H} / \mathrm{He}$ ion ratio of $\sim 20$ for $1-1.5 \mathrm{MeV} / \mathrm{n}$ particles in the heliosheath (Stone et al. 2007). These results are similar to the value obtained from HSTOF for the forward $\left(210^{\circ}-300^{\circ}\right)$ 


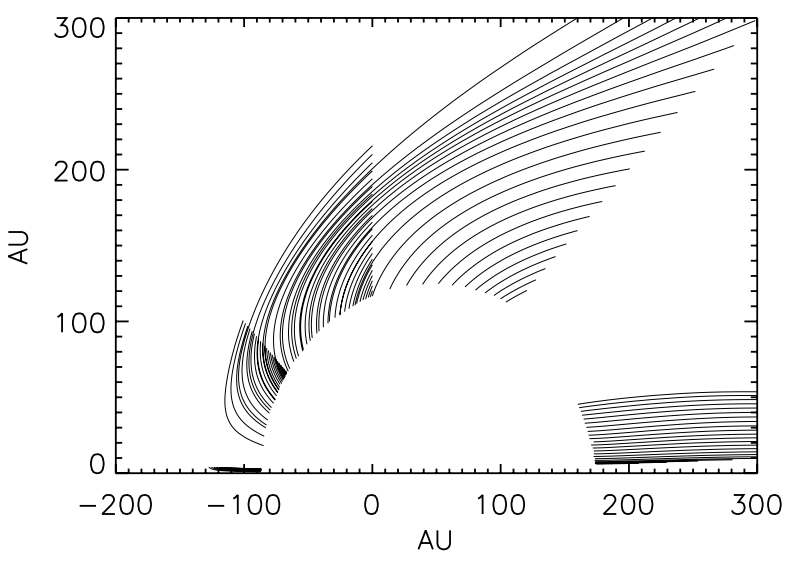

Fig. 3. Flow lines in the ecliptic plane from the termination shock to five selected lines of sight directed at the angle $\theta=1.8^{\circ}, 45^{\circ}, 90^{\circ}, 135^{\circ}$, and $178.2^{\circ}$ from the forward (upwind) direction.

longitude sector, although the uncertainty is high. The flank sectors have lower $\mathrm{H}^{+} / \mathrm{He}^{+}$, particularly the $1996-2010$ data.

\section{Calculating the heliosheath ion distributions}

\subsection{Model of the heliosphere}

The model of the heliosphere used in our simulations is based on the Bonn model (Fahr et al. 2000; Scherer \& Fahr 2003a). It consists of a numerical solution of the gas dynamics equations for five fluid components: the bulk plasma, the pick-up protons (with the flow velocity equal to the bulk plasma), the neutral hydrogen from the interstellar medium and the anomalous and galactic cosmic ray components, described by their pressure. The version that we used assumes the interstellar hydrogen density $0.2 \mathrm{~cm}^{-3}$ and electron density $0.04 \mathrm{~cm}^{-3}$; the shortest distance to the termination shock is $85 \mathrm{AU}$ and from the shock to the heliopause 42.5 AU. The flow is axisymmetric (with the symmetry axis parallel to the inflow of the interstellar medium), so that the asymmetries of the heliosphere caused by the solar wind structure or the interstellar magnetic field cannot be included. One consequence is that the difference in distances to the termination shock as observed by Voyager 1 and Voyager 2 is not reproduced by the model (see Fig. 7). Although the time-dependent (on the solar cycle scale) version is available, we used here only the time-stationary solution.

Figure 3 shows selected plasma flow lines in the inner heliosheath following from the model.

We extended the model by adding the solar magnetic field treated in the "kinematic" approximation: that is, we ignored the effect of the magnetic field on the plasma flow. The magnetic field was calculated from the "freezing-in" condition with the inner boundary condition provided by the Parker field. The field was used for modelling the parallel diffusion of the energetic particles.

We used a spherical system of coordinates $(r, \theta, \phi)$ centred at the Sun with the $z$ axis directed towards the apex of the interstellar medium inflow (the upwind direction). In the vicinity of the ecliptic plane (the HSTOF field of view) the angle $\theta$ is approximately equivalent to the (shifted) ecliptic longitude, with $\theta=0^{\circ}$ corresponding to the longitude $\sim 252^{\circ}$ of the apex.

The calculations are restricted to the region within $2000 \mathrm{AU}$ from the Sun, so that the more distant regions of the heliotail are not included. This cutoff affects the region $\theta \geq 165^{\circ}$.
The Bonn model was used as a basis for investigating the energetic particle distributions (Scherer \& Ferreira 2005; Ferreira et al. 2007a,b, 2008) and the production of ENA in the heliosphere (Scherer \& Fahr 2003b; Fahr \& Scherer 2004; Sternal et al. 2008), but none of these studies considered particles in the HSTOF energy range.

\subsection{Energetic particle spectrum at the termination shock}

We are interested in the energetic particles with energies close to the HSTOF energy range, which is much less than the typical anomalous or galactic cosmic ray energies. Calculations were made for a wider energy range $1-200 \mathrm{keV} / \mathrm{n}$, which permits a comparison with IBEX and INCA. We used a test particle approach and did not attempt to link the particle energy spectrum with the anomalous and galactic cosmic ray pressure calculated in the Bonn model.

The process of acceleration leading to the termination shock particle spectrum is not described by the model. Instead, we assumed the form of the energetic particle spectrum immediately downstream from the termination shock and used it as a boundary condition for the inner heliosheath. We did not include acceleration by the second-order Fermi process and the effects of the heliospheric current sheet. A concise theoretical review of acceleration and transport of the energetic particles (including the turbulence) in the heliosheath is given in Kallenbach et al. (2006). Models including the turbulence were studied by Chalov and Fahr (see Chalov et al. 2003).

We assumed that the energetic ion $\left(\mathrm{H}^{+}\right.$and $\left.\mathrm{He}^{+}\right)$distribution function at the termination shock in the energy range $\sim 10-200 \mathrm{keV} / \mathrm{n}$ is described by a simple power law in energy. This is in approximate agreement with the Voyager $1 Z \geq 1$ ion spectrum (Decker et al. 2005). To make a connection to lower energy we assumed that there is also a low-energy core component. It is constructed to satisfy the requirement (following from Voyager 2 observations) that most of the solar wind energy on crossing the termination shock is transferred to the pick-up ions. The form of the core (a simple Maxwellian) was chosen solely for simplicity.

The total distribution at the termination shock in the plasma frame is given by $(i=\mathrm{H}$ or $\mathrm{He})$ :

$$
\begin{aligned}
& f_{\mathrm{i}, \text { shock }}(\boldsymbol{r}, v)=C_{i} n_{\mathrm{PUI}, \mathrm{i}}(\boldsymbol{r})(E / n)^{-\delta-1} \quad\left(E>E_{m}\right) \\
& =n_{\text {core }, \mathrm{i}}(\boldsymbol{r}) \frac{\exp -\left(v / v_{T}\right)^{2}}{\pi^{3 / 2} v_{T}^{3}} \quad\left(E \leq E_{m}\right) \text {, }
\end{aligned}
$$

where $n_{\mathrm{PUI}, \mathrm{i}}$ is the corresponding pick-up ion density just upstream of the shock, $E / n$ the energy per nucleon corresponding to the velocity $v$ and $C_{i}$ a constant independent of the position on the shock. We used $\delta=1.65$ (flux $\propto E^{-1.65}$ ). In the Maxwellian term $n_{\text {core, } i}$ is the core particle number density immediately downstream from the shock and $v_{\mathrm{T}}$ their thermal speed. The power law distribution is applicable down to the "merging energy" $E_{\mathrm{m}}$ at which it is equal to the assumed core distribution.

To fix $C_{\mathrm{H}}$ we fitted the proton flux following from the powerlaw formula from Eq. (1) (with $\boldsymbol{r}$ the $\theta=0^{\circ}$ point on the shock surface) to the Voyager $1 Z \geq 1$ ion flux taken from Fig. 3 of Krimigis et al. (2010). The resulting energetic proton spectrum (including also the core part) at the $\theta=0^{\circ}$ point on the termination shock is shown in Fig. 4 together with the Voyager 1 and Voyager 2 ion spectra.

$C_{\mathrm{He}}$ was obtained by requiring $\mathrm{H}^{+} / \mathrm{He}^{+}$flux ratio to be 20 at the termination shock in the Voyager 1 direction. This choice 


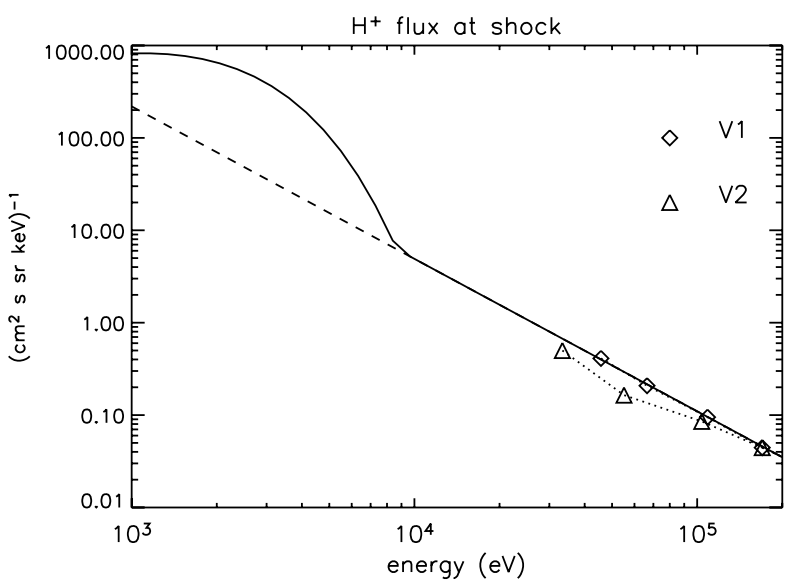

Fig. 4. Proton flux spectrum at the termination shock (at the point $\theta=0^{\circ}$ ) assumed in the model. The dashed line shows the pure powerlaw case. The symbols show $Z \geq 1$ Voyager 1 and 2 heliosheath ion spectra taken from Krimigis et al (2010).

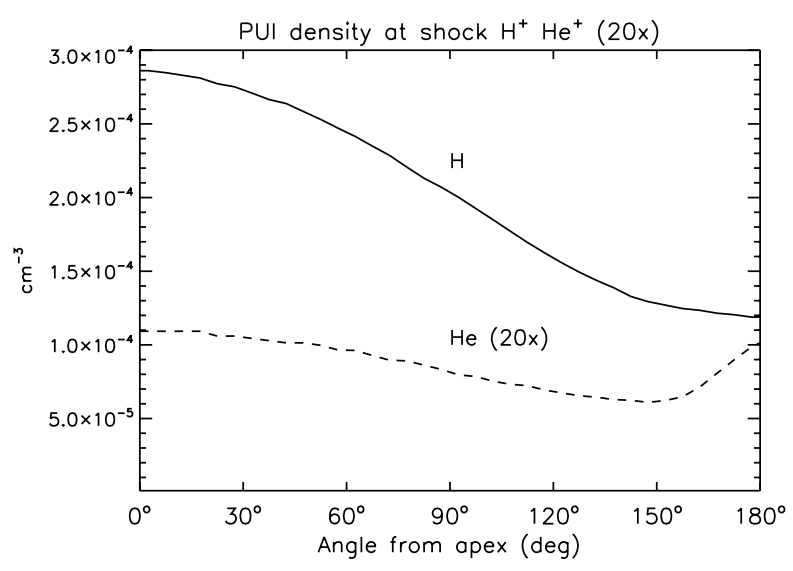

Fig. 5. Density distributions of the pick-up protons and $\mathrm{He}^{+}$ions (the latter multiplied by 20) immediately upstream of the termination shock assumed in the calculations.

was motivated by observations (Decker et al. 2005; Stone et al. 2007; see also the discussion in Sect. 2).

The parameters of the core (the thermal speed $v_{\mathrm{T}}$ and the number density $\left.n_{\text {core }, \mathrm{i}}(\boldsymbol{r})\right)$ were obtained from two requirements: (1) pick-up ion number conservation across the shock and (2) the total energy transferred to the pick-up ions equals 0.8 the solar wind energy upstream of the shock (Richardson et al. 2008a). The pre-shock solar wind parameters and the pick-up ion number densities were taken from the Bonn model.

A qualitatively similar core + tail distribution was obtained by Giacalone (Giacalone 2005; Giacalone \& Decker 2010) from numerical simulation of the acceleration process at the termination shock. The core distribution of Giacalone is less wide than our Maxwellian, so that our "merging energy" is higher ( $\sim 10 \mathrm{keV}$ compared to his $\sim 5 \mathrm{keV}$ ).

The pick-up ion densities immediately upstream of the shock used in the calculation are shown in Fig. 5 as functions of the angle $\theta$. The proton distribution was taken from the Bonn model. We used a separate model of the interstellar helium distribution (Bzowski, priv. comm.) in the heliosphere to calculate the pickup helium distribution.

\subsection{Particle distributions in the heliosheath}

We calculated the energetic particle distribution downstream from the termination shock by solving approximately the timestationary Parker equation

$\left[-\boldsymbol{V} \cdot \nabla-\beta_{c x}+\frac{1}{3}(\nabla \cdot \boldsymbol{V}) \frac{\partial}{\partial \log v}+\nabla \cdot D \cdot \nabla-\beta_{\mathrm{esc}}\right] f=0$.

Here $\boldsymbol{V}(\boldsymbol{r})$ is the plasma flow velocity (we neglected the drifts), $D$ is the spatial diffusion tensor, $\beta_{\mathrm{cx}}$ the loss rate to neutralization by charge-exchange and $\beta_{\text {esc }}$ the loss rate due to escape through the heliopause. The distribution function $f(\boldsymbol{r}, v)$ we assumed to be isotropic in velocity in the plasma frame. Charge-exchange cross sections were calculated using the fits from Barnett et al. (1990). For the $\mathrm{H}+\mathrm{H}^{+}$charge exchange cross section the difference between Barnett et al. and the more recent fit (Lindsay \& Stebbings 2005) is of the order of few percent except for the energy region between $\sim 10$ and $\sim 70 \mathrm{keV}$ where the Lindsay \& Stebbings cross section is larger by up to $30 \%$ (at $30 \mathrm{keV}$ ) and by $15-20 \%$ in the HSTOF energy range.

The ordering of terms in Eq. (2) corresponds to successive approximations (1)-(5) that we used to estimate the effect of different processes. In the approximation (1) the equation reduces to the first term $(\boldsymbol{V} \cdot \nabla f=0$, describing convection). The next approximation (2) adds the charge-exchange neutralization loss, (3) the adiabatic acceleration/deceleration, (4) the parallel diffusion in space and (5) escape across the heliopause.

In the approximations (1) to (3) the solution to Eq. (2) can be obtained by solving an ordinary differential equation along the plasma flow line $\boldsymbol{r}(s)$. For example, in the approximation (3) the solution can be written as

$f^{(3)}=F(\boldsymbol{r}(s), v(s)) \exp \left[-\int_{0}^{s} \mathrm{~d} s^{\prime} \frac{\beta_{\mathrm{cx}}\left(s^{\prime}\right)}{V\left(\boldsymbol{r}\left(s^{\prime}\right)\right)}\right]$,

where $v$ is the particle speed in the plasma frame, $V \mathrm{~d} \boldsymbol{r}(s) / \mathrm{d} s=$ $\boldsymbol{V}, V \mathrm{~d} \log v(s) / \mathrm{d} s=-(1 / 3)(\nabla \cdot \boldsymbol{V}), \beta_{\mathrm{cx}}$ is the ion neutralization rate dominated by charge exchange and $s$ is the length parameter along the flow line. $F$ is determined by the boundary condition at the termination shock where $s=0$. The approximation (2) corresponds to $\mathrm{d} \log v / \mathrm{d} s=0$ and the approximation (1) also to $\beta_{\mathrm{cx}}=0$.

The diffusion and the escape processes were treated using a very simplified approach.

The effect of parallel diffusion on the energetic ion distribution $f(\boldsymbol{r}, v)$ at the point $\boldsymbol{r}$ was estimated as follows. First, the distributions in the approximation (3) were calculated for a set of points along the magnetic field line $\boldsymbol{r}_{B}(s)$ passing through $\boldsymbol{r}$ ( $s$ is the length parameter along this field line). The distribution in the approximation (4) (including parallel diffusion) is then given by

$f^{(4)}\left(\boldsymbol{r}_{B}(s), v\right)=\int \mathrm{d} s^{\prime} \frac{\exp \left[-\left(s-s^{\prime}\right)^{2} / 4 D(v) t\left(s^{\prime}\right)\right]}{\left(4 \pi D(v) t\left(s^{\prime}\right)\right)^{1 / 2}} f^{(3)}\left(\boldsymbol{r}_{B}\left(s^{\prime}\right), v\right)$,

where $t\left(s^{\prime}\right)$ is the flow time from the shock and $f^{(3)}\left(s^{\prime}, v\right)$ the ion distribution obtained in the approximation (3) at the point $s^{\prime}$ on the magnetic field line. The parallel diffusion coefficient $D(v)$ we defined as $D(v)=(1 / 3) v \Lambda$, with the mean free path $\Lambda$ assumed to be a constant. In most calculations we used $\Lambda=3 \mathrm{AU}$ (derived from the phenomenological formula of le Roux et al. 1996).

Our treatment of parallel diffusion is clearly very approximate. We described the spreading of the particle distribution 
along the magnetic field line as a one-dimensional random walk. We disregarded the change in the diffusion coefficient $D$ on the way from the termination shock to the point at which the distribution is calculated. Also, in calculating the effects of adiabatic acceleration and of the loss terms we neglected the fact that the true path of the diffusing particle is different from the plasma flow line.

The escape process is described by the loss term $\beta_{\text {esc }} \equiv$ $1 / \tau_{\text {esc }}(\boldsymbol{r}, v)$ where the characteristic time for the escape for a particle with the velocity $v$ is taken to be $\tau_{\mathrm{esc}}(\boldsymbol{r}, v)=l_{\mathrm{HP}}(\boldsymbol{r})^{2} / D_{\mathrm{T}}$ with $l_{\mathrm{HP}}(\boldsymbol{r})$ the distance from $\boldsymbol{r}$ to the heliopause and $D_{\mathrm{T}}=(1 / 3) v \Lambda_{\mathrm{T}}$. This escape time corresponds to a random walk over the distance $l_{\mathrm{HP}}$ with the mean free path $\Lambda_{\mathrm{T}}$. That is, we assumed that the escape can be described as diffusion in space, with the parameters $D_{\mathrm{T}}$ and $\Lambda_{\mathrm{T}}$ different from those for parallel diffusion. $\Lambda_{\mathrm{T}}$ is assumed to be a constant and set to $0.1 \mathrm{AU}$ in most of the calculations. Since the escape process is likely to involve moving across the heliospheric magnetic field, we expect $\Lambda_{\mathrm{T}}$ to be smaller than the parallel mean free path $\Lambda$.

The ion distributions provide a source for the ENA flux. The ENA flux $J_{\text {ENA }}$ from a given direction we calculated as

$$
\begin{aligned}
J_{\mathrm{ENA}}(E)= & \int \mathrm{d} s J_{\text {ion }}(E) \frac{v_{\text {rel }}}{v}\left[\sigma_{\mathrm{cx}, \mathrm{H}}\left(E_{\mathrm{rel}}\right) n_{\mathrm{H}}\right. \\
& \left.+\sigma_{\mathrm{cx}, \mathrm{He}}\left(E_{\mathrm{rel}}\right) n_{\mathrm{He}}\right] \mathrm{e}^{-\Delta}
\end{aligned}
$$

where $J_{\text {ion }}(E)$ is the sunward-directed parent ion flux in the observer frame calculated in the model, $v$ the speed of the ENA particle of energy $E, v_{\text {rel }}=\left|\boldsymbol{v}-\boldsymbol{v}_{\mathrm{H}}\right|$ the velocity relative to the neutral gas background, $E_{\text {rel }}$ the energy corresponding to $v_{\text {rel }}, n_{\mathrm{H}}$ and $n_{\mathrm{He}}$ the densities of the background neutrals and $\Delta$ the ENA loss. The ENA loss is negligible in the HSTOF energy range. The integral over the distance $s$ runs over the segment of the line-of-sight within the inner heliosheath (between the termination shock and the heliopause).

\section{Results from the model: ion distributions}

We used the model to calculate the energetic ion distributions in the inner heliosheath (between the termination shock and the heliopause) along selected directions (lines of sight) that are constrained to the ecliptic plane and specified by the angle $\theta$ counted from the inflow direction.

Figure 6 shows the calculated energetic proton flux intensity as a function of distance from the Sun along two directions: the forward (upwind, or the interstellar matter inflow) direction $\left(\theta=0^{\circ}\right)$ and the crosswind direction in the ecliptic plane $\left(90^{\circ}\right)$. The flux is sunward-directed, converted from the plasma frame (where it is isotropic) to the observer frame. The energy (66 keV, which is in the HSTOF range) refers to the observer frame.

The results of successive approximations (1) to (5) are shown. Each approximation differs from the previous one by including one additional process.

In the approximation (1) the energetic ion distribution function in the plasma frame does not change along the flow line. The variation seen in Fig. 6 has two causes. The energetic ions arriving at the lines of sight corresponding to smaller $\theta$ are convected from the parts of the shock (Fig. 3) where the pick-up proton density (and the energetic ion density) is higher (Fig. 5). Also, the plotted flux is in the observer frame and therefore affected by the local plasma speed along the line of sight, which decreases with distance.

Neutralization loss (approximation (2)) has a considerable effect by reducing the energetic ion flux, particularly at longer
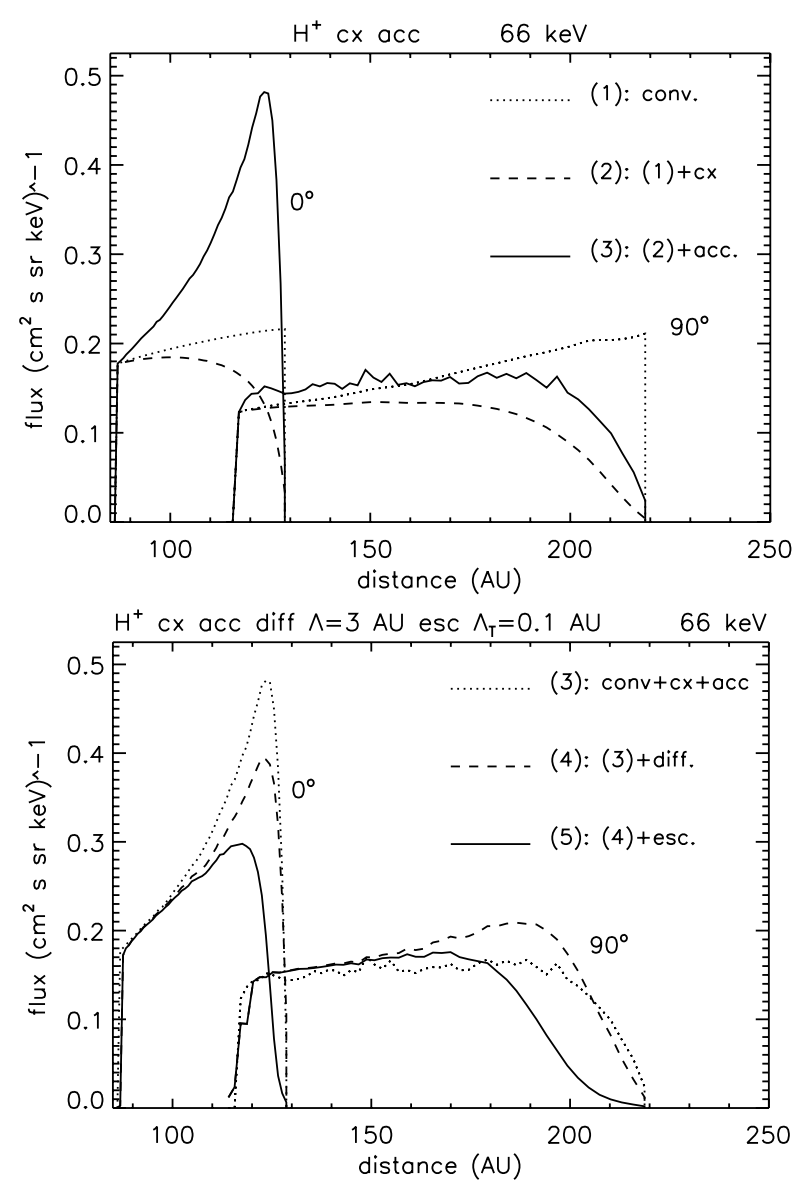

Fig. 6. Calculated fluxes of $66 \mathrm{keV}$ protons (the energy in the fixed frame) in the inner heliosheath as a function of distance from the Sun. The profiles are shown for two directions: forward (upwind, $\theta=0^{\circ}$ ) and the flank (crosswind, $\theta=90^{\circ}$ ) in the ecliptic plane. The upper figure shows the results from the approximations (1) (convection only), (2) (charge-exchange neutralization loss added) and (3) (adiabatic acceleration added). The lower figure shows the approximations (3), (4) (parallel diffusion added, mean free path $\Lambda=3 \mathrm{AU}$ ) and (5) (escape across the heliopause added, mean free path $\Lambda_{\mathrm{T}}=0.1 \mathrm{AU}$ ).

distances (long convection time). Adiabatic acceleration is important near $\theta=0^{\circ}$. In Czechowski et al. (2006b, 2010), where preliminary results of our calculations were presented, the effect of adiabatic acceleration was overestimated by a large factor because of an error in the code. This led to steep spatial gradients and strong spatial diffusion effects.

Parallel diffusion (approximation (4)) moves the particles along the magnetic field lines away from the high-flux regions and towards the low-flux regions. The effect is moderate (10-20 percent change in the flux). Escape across the heliopause (approximation (5)) reduces the ion flux at long distances. Despite a low value of the mean free path assumed (0.1 AU) this effect is visible already at the distance of 10 to few tens AU from the heliopause. We interpret this as caused by the slowing down of the plasma flow near the heliopause, so that the slow process of perpendicular diffusion for the convected energetic ions has enough time to operate.

The energetic ion flux profiles in the heliosheath are now partly known from Voyager measurements. In the low-energy channels (which overlap with the HSTOF energy channels) the flux is approximately constant with distance from the termination shock except for transient effects. In Fig. 7 we compare the 


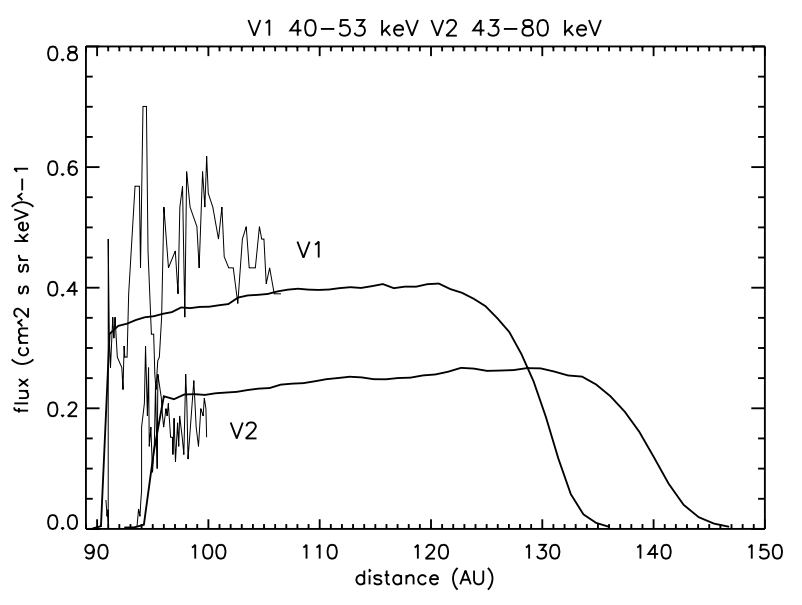

Fig. 7. Calculated energetic proton fluxes along the trajectories of Voyager 1 (40-53 keV protons) and Voyager 2 (43-80 keV protons) in the heliosheath compared with the Voyager LECP measurements up to 2009/105 (taken from Hsieh et al. 2010). The Voyager data are shifted in distance to fit the the termination shock position in the Bonn model.

Voyager 1 and Voyager $2 Z \geq 1$ heliosheath ion fluxes in the LECP energy bins close to the HSTOF energy range (40-53 keV for Voyager 1 and 43-80 keV for Voyager 2) with the $\mathrm{H}^{+}$flux calculated in the model along the Voyager 1 and Voyager 2 trajectory directions. With our choice for $C_{\mathrm{H}}$ the calculated fluxes are within 20 percent from the observed fluxes averaged over the distance. The calculated flux increases initially with distance, but the increase is slow.

The model predicts a falloff in the ion flux within about 10 AU from the boundary caused by the escape through the heliopause. This imitates the "transition layer" suggested by Suess (1990). A flux decrease was observed in the Voyager 1 data (Krimigis et al. 2011) by about $27 \%$ during last three months of 2010 and it was suggested (Krimigis et al. 2011) that it may be a signature of the transition layer. If so, the heliosheath in the Voyager 1 direction would be much thinner than in our model and more in line with HSTOF results. However, it is also possible that the observed falloff is caused by solar cycle effects on the heliosheath (Krimigis et al. 2011).

\section{Results from the model: the ENA fluxes}

Using the Bonn model for the heliosheath and the ion fluxes following from our model we calculated the ENA fluxes along different lines of sight for an observer situated at $1 \mathrm{AU}$ from the Sun. The main results are:

(1) The direction dependence of the ENA flux is different at different energies.

(2) The HSTOF flux is significantly lower than the model prediction, but shows a similar dependence on direction.

In Fig. 8 we show how the energy dependence of the directional structure of the hydrogen ENA flux emerges in our model. In approximation (1) (convection only) the tail/flank/forward flux ratio is approximately the same at all energies except for the core region $(E<10 \mathrm{keV})$. In the approximation (2) the parent ion charge-exchange neutralization loss causes a downward shift of the ENA spectra that is larger at low energy (due to larger charge-exchange cross section) and depends on the direction of observation because of flow time effects. In consequence, the flux from the tail becomes less than the flux from the forward
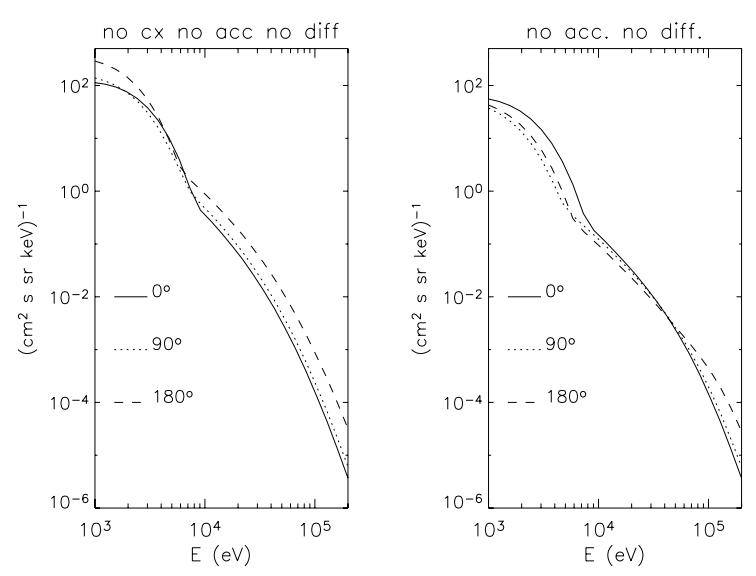

Fig. 8. ENA H energy spectra obtained from the model ion distributions in approximations (1) and (2) for 3 directions: $\theta=0^{\circ}$ (forward) $90^{\circ}$ (crosswind), and $180^{\circ}$ (the heliotail). In approximation (1) (convection only) the energy dependence of the directional structure of the ENA flux results mainly from the $\theta$-dependence of the ion core spectrum at the termination shock. In approximation (2) the charge exchange neutralization of the parent ions causes an energy-dependent shift in the ENA spectrum. The result, with the crossover of the heliotail and the forward spectra at $\sim 40 \mathrm{keV}$, is not changed much by subsequent approximations.

sector below a crossover energy of about $40 \mathrm{keV}$. This implies that HSTOF can observe a different dependence on direction of the hydrogen ENA flux than the lower energy instruments INCA (highest energy channel $\sim 46 \mathrm{keV}$ : Krimigis et al. 2009) and $\operatorname{IBEX}(E \leq 6 \mathrm{keV}$ : McComas et al. 2009) The result is not substantially changed by taking adiabatic acceleration, diffusion and escape processes into account. Our preliminary result (Czechowski et al. 2006b), which suggested a strong diffusion effect, was affected by the error in calculating the adiabatic acceleration (see the remark in Sect. 4).

Figure 9 shows the calculated hydrogen and helium ENA spectra for five directions in the HSTOF energy range compared with the HSTOF 1996-2010 data for one of the flank sectors $\left(300^{\circ}-30^{\circ}\right)$. The HSTOF hydrogen (helium) data points are below the model ENA spectrum by a factor $>6(\sim 3)$. This difference between $\mathrm{H}$ and $\mathrm{He}$ can be understood as due to the $\mathrm{H} / \mathrm{He}$ ratio of 20 assumed in the model, while the HSTOF data for the flanks correspond instead to $\mathrm{H} / \mathrm{He}=10$. The energy dependence of the directional distribution is also different for the $\mathrm{H}$ and He cases: for hydrogen, the flux from the tail direction dominates at high energy (above $\sim 40 \mathrm{keV}$ ) but for lower energy the forward direction becomes dominant. For helium, the flux from the tail direction stays dominant in the whole energy range. The main reason is that the $\mathrm{He}^{+}$pick-up ion density at the termination shock is (in contrast to $\mathrm{H}^{+}$) not suppressed in the heliotail region (Fig. 5).

The similarity between the directional dependence of the model ENA flux and the HSTOF data is illustrated in Fig. 10, where the model results are compared with the HSTOF data for the 1996-2005 period (the lowest energy bins) from four longitude sectors. The model flux is scaled down by $1 / 4.5(\mathrm{H})$ and $1 / 2.5(\mathrm{He})$. The highest flux is coming from the heliotail direction $\left(\theta \sim \pm 180^{\circ}\right)$ while the flux from the flanks $\left(\theta \sim 90^{\circ}\right.$ and $\left.\theta \sim-90^{\circ}\right)$ is comparable to that from the forward direction $\left(\theta \sim 0^{\circ}\right)$ despite the greater thickness of the heliosheath in the flank sectors.

The main reason for this behaviour of the ENA flux can be deduced from Fig. 11, which shows the directional dependence 

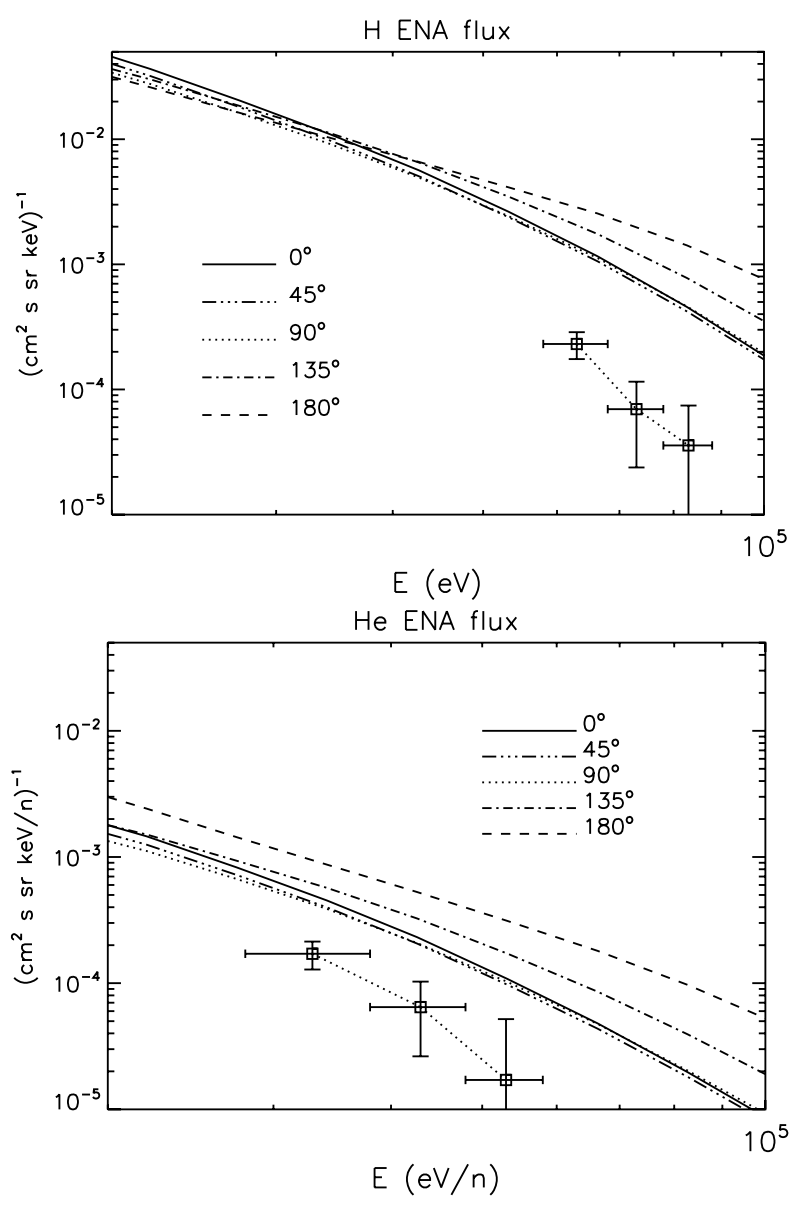

Fig. 9. ENA $\mathrm{H}$ and $\mathrm{He}$ energy spectra obtained from the model ion distributions (approximation (5)) for five directions: $\theta=0^{\circ}$ (forward), $45^{\circ}$, $90^{\circ}$ (crosswind), $135^{\circ}$ and $180^{\circ}$ (the heliotail). The HSTOF 1996-2010 ENA $\mathrm{H}$ and $\mathrm{He}$ flux data (the $300^{\circ}-30^{\circ}$ ecliptic longitude sector) are shown for comparison.

of the ENA hydrogen flux at $66 \mathrm{keV}$ calculated in different approximations. In the approximation (1) (convection only) the calculated ENA flux from the flanks is much higher than from the forward direction and increases further towards the tail up to $\theta \sim 150^{\circ}$ (the falloff beyond this angle is not caused by the calculation region cutoff at $2000 \mathrm{AU}$, which affects the results only for $\theta>165^{\circ}$ ). Including the charge-exchange loss (approximation (2)) leads to a substantial reduction in the energetic ion flux and consequently in the ENA flux from the flank sector, because of the long convection time of the parent ions from the termination shock. In result, the ENA flux from the flank sectors in approximation (2) is only $\sim 1.5$ higher than from the forward sector.

\section{Discussion}

In Fig. 12 the HSTOF hydrogen data (forward sector) and our model calculations are compared with the recently published ENA data from two dedicated ENA instruments: IBEX Hi (Schwadron et al, 2011: non-ribbon only, four directions: forward, heliotail, west flank and east flank) and INCA (Krimigis et al. 2010: the "minimum" flux and two Voyager directions). The model flux is shown for three directions: $\theta=0^{\circ}, 90^{\circ}$ and $180^{\circ}$. Compared with IBEX, the model flux has the same order of magnitude (with no adjustment of parameters) but it is clear that our simple assumption about the form of the core does
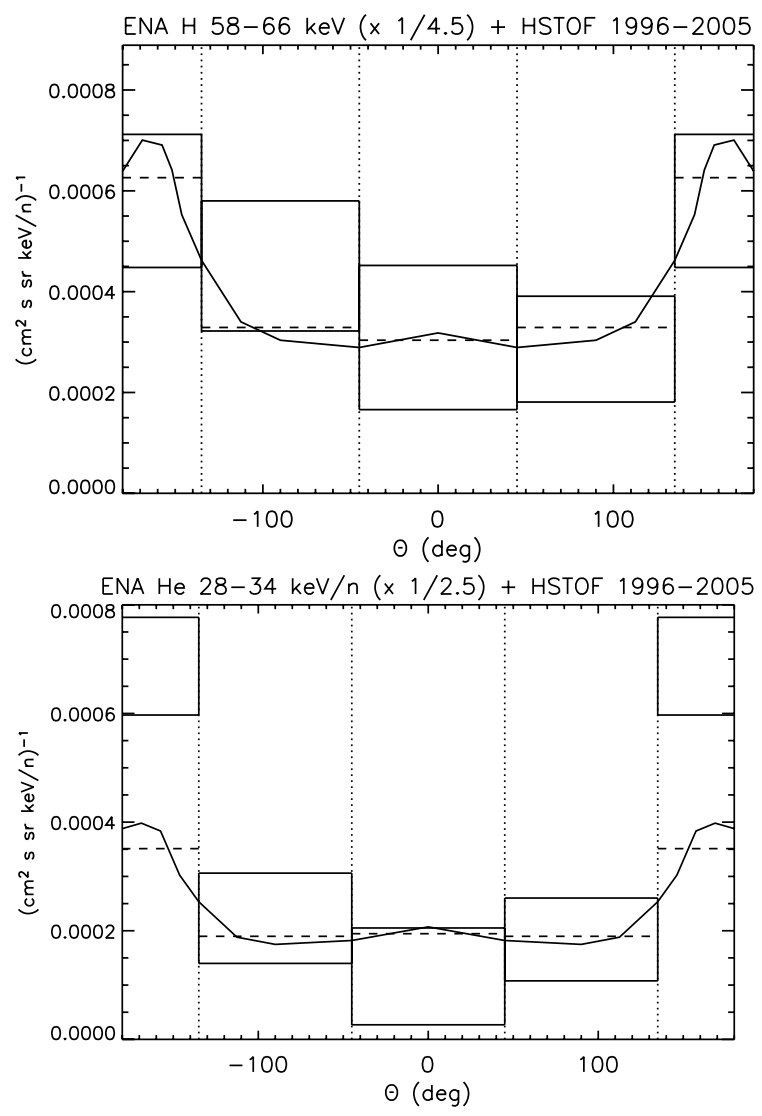

Fig. 10. Directional dependence of the model hydrogen and helium ENA flux (solid line) compared with the HSTOF 1996-2005 data for four ecliptic longitude sectors (boxes). The HSTOF data correspond to the lowest energy bins (58-68 keV for hydrogen, $28-38 \mathrm{keV} / \mathrm{n}$ for helium). The height of the boxes is the same as the height of the error bars in Figs. 1, 2 (the lowest energy data points for 1996-2005). The model ENA flux is averaged over similar energy intervals $(58-66 \mathrm{keV}$ for $\mathrm{H}, 28-34 \mathrm{keV}$ for $\mathrm{He}$ ) and scaled down by the factors $1 / 4.5(\mathrm{H})$ and $1 / 2.5(\mathrm{He})$. The averages of the model flux over the ecliptic longitude sectors are shown by dashed lines.

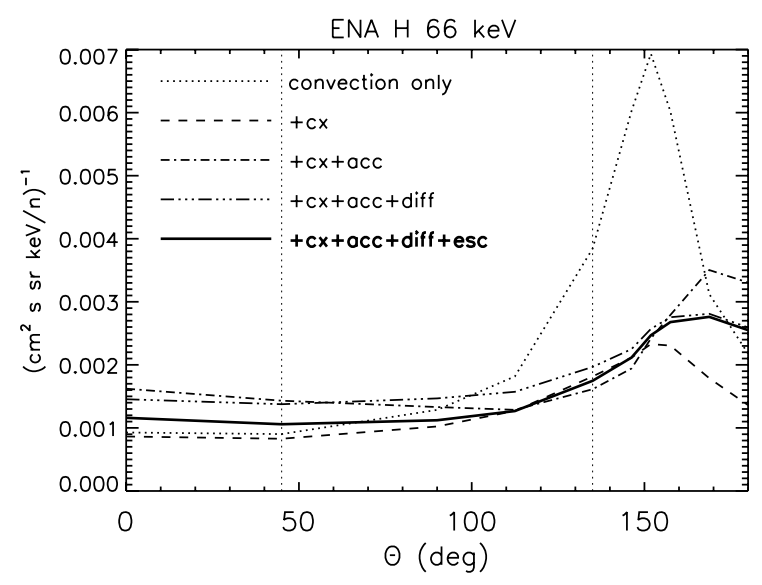

Fig. 11. Model ENA $66 \mathrm{keV}$ hydrogen flux as a function of direction $\Theta$ (counted from the forward direction), in the approximations (1) to (5). Dotted vertical lines show the limits of the flank sector.

not reproduce the IBEX ENA spectrum and its direction dependence. The INCA data at lower energy run above our model results suggesting perhaps a need of a wider core. The HSTOF hydrogen ENA flux is significantly below the model result and 


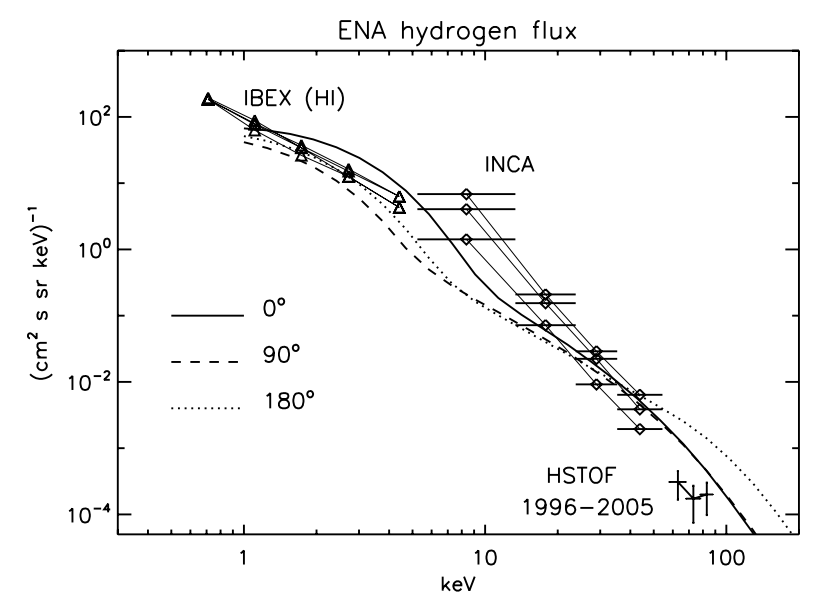

Fig. 12. Model H ENA energy spectrum for three directions $\left(\theta=0^{\circ}\right.$ solid line, $90^{\circ}$ dashed, $180^{\circ}$ dotted) compared with the ENA data from IBEX (Hi), INCA and HSTOF (1996-2005, forward sector).

Table 2. $L_{\mathrm{eff}}$ derived from observations (HSTOF ENA and Voyager ion data).

\begin{tabular}{lcc}
\hline \hline Sector & $\begin{array}{c}L_{\mathrm{eff}}(\mathrm{V} 1) \\
(\mathrm{AU})\end{array}$ & $\begin{array}{c}L_{\mathrm{eff}}(\mathrm{V} 2) \\
(\mathrm{AU})\end{array}$ \\
\hline $1996-2005$ & & \\
$210^{\circ}-300^{\circ}$ & $19.9 \pm 6.2$ & $31.0 \pm 9.5$ \\
$300^{\circ}-30^{\circ}$ & $13.8 \pm 4.7$ & $20.8 \pm 7.3$ \\
$120^{\circ}-210^{\circ}$ & $24.2 \pm 5.7$ & $37.3 \pm 8.7$ \\
\hline $1996-2010$ & & \\
$300^{\circ}-30^{\circ}$ & $11.2 \pm 2.5$ & $17.0 \pm 3.9$ \\
$120^{\circ}-210^{\circ}$ & $10.2 \pm 2.6$ & $16.1 \pm 4.0$ \\
\hline
\end{tabular}

(if we use 1996-2005 data) lies close to the lower limit of the extrapolated INCA flux.

The implication of the low ENA flux for the size of the heliosphere is illustrated in Table 2, which shows the effective thickness of the heliosheath $L_{\mathrm{eff}}$, a rough estimation of the actual thickness $L$ under assumption that the parent ion flux is given by the Voyager measurement. This method of estimating $L$ was used by different authors (Czechowski et al. 2006a, 2008; Hsieh et al. 2010; Krimigis et al. 2009, 2010). The values in Table 2 were obtained by fitting the H HSTOF ENA data using $J_{\mathrm{ENA}}=J_{\text {ion }} \sigma_{\mathrm{cx}, \mathrm{H}} L_{\mathrm{eff}} n_{\mathrm{H}}$ with $J_{\text {ion }}(E)$ equal to the $Z \geq 1$ ion flux measured by Voyager $1\left(L_{\mathrm{eff}}(V 1)\right)$ or Voyager $2\left(L_{\mathrm{eff}}(V 2)\right)$ and taking $n_{\mathrm{H}}=0.1 \mathrm{~cm}^{-3}$ (Bzowski et al. 2008; Richardson et al. 2008b; Pryor et al. 2008).

Figure 13 shows the model prediction for the effective thickness $L_{\text {eff }}$ and the actual thickness $L$ as a function of direction (in the ecliptic plane, at the angle $\theta$ from the inflow direction). The effective thickness is defined similarly as in Table 2 (see the previous paragraph) but, instead of the ENA flux observed by HSTOF and the ion flux measured by Voyagers, we used the model predictions. $J_{\mathrm{ENA}}$ is the H ENA flux (from the direction $\theta$, at the energy $66 \mathrm{keV}$ ) calculated in the model. $J_{\text {ion }}$ is the model-calculated proton flux (averaged over the distance $30 \mathrm{AU}$ downstream from the termination shock) at the same energy, coming from the direction corresponding to the Voyager 1 trajectory. Figure 13 implies that (1) $L_{\text {eff }}$ is a reasonable approximation to $L$ in the forward sector, but not in the flanks, and (2) the values of $L_{\text {eff }}$ in the forward sector and in the flanks are close to each other.

The values of $L_{\mathrm{eff}}$ predicted by the model (Fig. 13) are higher than the values following from the HSTOF observations

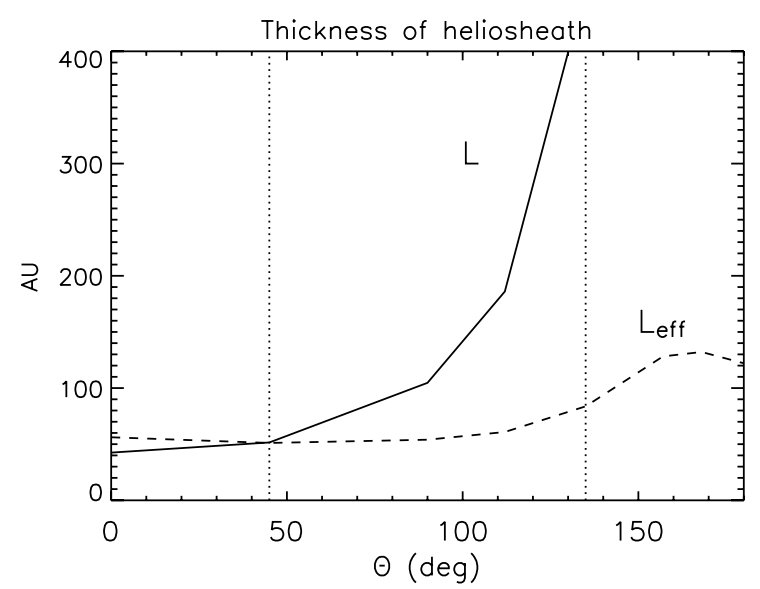

Fig. 13. Effective thickness $L_{\text {eff }}$ and the actual thickness of the heliosheath $L$ calculated in the model described in Sect. 3. The angle $\theta$ is counted from the forward direction (upwind, or apex direction of the interstellar medium inflow). Note the difference between the $L_{\mathrm{eff}}$ shown in the figure (based on model calculations) and the $L_{\text {eff }}$ in Table 2 (derived from observations).

(Table 2). The reason is that the ENA flux calculated in the model is higher than that measured by HSTOF (Fig. 9).

The 1996-2005 HSTOF data for the forward sector indicate a thickness of $\sim 20-30$ AU (Table 2). Figure 13 suggests that $L_{\mathrm{eff}}$ for the flank sectors can still be used as a rough estimate of the thickness of the forward sector. The 1996-2005 HSTOF data for the flanks give a similar range of values (14-37 AU) as for the forward sector, but the 1996-2010 data indicate the thickness of only $10-17 \mathrm{AU}$.

The values of $L_{\text {eff }}$ given in Table 2 are significantly lower than in Czechowski et al. (2006a, 2008). The reasons are: the average Voyager ion flux used here is higher than the early measurements near the shock; the $Z \geq 1$ ion flux is interpreted as protons (in Czechowski et al. 2006, 2008, only 2/3 of it was assumed to be protons). The result for the forward sector is close to that of Hsieh et al. (2010). $L_{\mathrm{eff}}$ for the 1996-2010 period are lower than for 1996-2005 because of the drop in the observed ENA hydrogen flux.

\section{Conclusions}

We presented the new ENA energy spectra (including the HSTOF data obtained in the period 1996-2010) for the flank sectors. The hydrogen ENA flux in the ecliptic longitude sector $120^{\circ}-210^{\circ}$ is lower by a factor of $\sim 2$ compared to that derived from the observations for the period 1996-2005. There is no such decrease in the He ENA flux. The H/He ratio in the flank sectors is $\sim 9$.

To interpret the ENA observations in terms of the heliosheath structure, we have developed a model of the energetic ion distribution in the inner heliosheath based on the Bonn model of the heliosphere. Although the model is very simplified (it is based on the time-stationary and axisymmetric model of the heliosphere), it illustrates the mechanisms shaping the ion distributions and therefore determining the ENA production rates.

The model predicts that the directional dependence of the ENA flux should not follow the variation in the thickness of the inner heliosheath (which steeply increases away from the forward direction). Instead, the calculated ENA flux from the flanks is close to that from the forward (upwind) sector. This agrees with the observations by HSTOF. 
At the same time, the ENA flux intensity predicted by the model is for all directions significantly higher than that measured by HSTOF.

One possible explanation is that the thickness of the heliosheath is smaller than the model predictions. The HSTOF results for the period 1996-2005 imply the thickness (in the forward sector) of $\sim 20-30$ AU, similar to the result of Hsieh et al. (2010) (21-28 AU) and of Krimigis et al. (2011) (27 AU for Voyager 1 direction). A similar distance to the heliopause (112-128 AU) was obtained in a semiempirical model by Webber and Intriligator (2011). One implication is that Voyager 1 should be already close to the outer boundary of the heliosphere. Low $L_{\text {eff }}$ values (10-17 AU) obtained from the HSTOF data for the period 1996-2010 in the flank sectors may perhaps be caused by the asymmetry of the heliosphere or time-dependent effects.

Acknowledgements. We thank Klaus Scherer for providing us with the results of his numerical model of the heliosphere and Maciej Bzowski for the model of the neutral helium distribution. A.C. acknowledges support from Polish Ministry of Science and Higher Education grant NS-1260-11-09.

\section{References}

Baranov, V., \& Malama Y. 1993, J. Geophys. Res., 98, 15157

Barnett, C. F., Hunter, H. T., Kirkpatrick, M. I., Alvarez, I., \& Phaneuf, R. A. 1990, Atomic Data for Fusion, Collisions of H, H2, He and Li Atoms and Ions with Atoms and Molecules, Oak Ridge Natl. Lab. Report, ORNL-6086VI, Oak Ridge, Tenn.

Bzowski, M., Möbius, E., Tarnopolski, S., Izmodenov, V., \& Gloeckler, G. 2008, A\&A, 491, 7

Chalov, S. V., Fahr, H. J., \& Izmodenov, V. V. 2003, J. Geophys. Res., 108, 1266

Czechowski, A., Hilchenbach, M., Hsieh, K. C., Kóta, J., \& Kallenbach, R. 2006a, ApJ, 647, L69

Czechowski, A., Hilchenbach, M., \& Kallenbach, R. 2006b, in The Physics of the Heliospheric Boundaries, ed. V. V. Izmodenov, \& R. Kallenbach, ISSI Scientific Report SR-005, Chap. 10, 311

Czechowski, A., Hilchenbach, M., Hsieh, K. C., Grzedzielski, S., \& Kóta, J. 2008, A\&A, 487, 329

Czechowski, A., Hilchenbach, M., Hsieh, K. C., \& Grzedzielski, S. 2010, in Pickup ions throughout the heliosphere and beyond, Proc. 9th Annual International Astrophysics Conference, Maui, Hawaii, 14-19 March, ed. J. le Roux, V. Florinski, G. P. Zank, \& A. J. Coates, AIP Conf. Proc., 1302, 104 Decker, R. B., Krimigis, S. M., Roelof, E. C., et al. 2005, Science, 309, 2020

Decker, R. B., Krimigis, S. M., Roelof, E. C., et al., 2008, Nature, 454, 67

Fahr, H. J., \& Scherer, K. 2004, Astra, 1, 3

Fahr, H. J., Kausch, T., \& Scherer, K. 2000, A\&A, 357, 268

Fahr, H. J., Fichtner, H., \& Scherer, K. 2007, Rev. Geophys., 45, 4003

Ferreira, S. E. S., Potgieter, M. S., \& Scherer, K. 2007a, J. Geophys. Res., 112, A11101, 357

Ferreira, S. E. S., Potgieter, M. S., \& Scherer, K. 2007b, ApJ, 659, 1777

Ferreira, S. E. S., Potgieter, M. S., \& Scherer, K. 2008, in Particle Acceleration and Transport in the Heliosphere and Beyond: 7th Annual Astrophysics Conference, ed. G. Li, Q. Hu, O. Verkhoglyadova, G. P. Zank, R. P. Lin, \& J. Luhmann (AIP), 355
Giacalone, J. 2005, ApJ, 628, L37

Giacalone, J., \& Decker, R. B. 2010, ApJ, 710, 91

Hilchenbach, M., Hsieh, K. C., Hovestadt, D., et al. 1998, ApJ, 503, 916

Hilchenbach, M., Hsieh, K. C., Hovestadt, D., et al. 2001, in The Outer Heliosphere: The New Frontiers, COSPAR Colloq. Ser., 11, 273

Hilchenbach, M., Kallenbach, R., Hsieh, K. C., \& Czechowski, A. 2006, in Physics of the heliosphere: a 10 year retrospective, Proc. 10th Annual International Astrophysics Conference, March 13-18, 2011 Maui, Hawaii, in press

Hovestadt, D., Hilchenbach, M., Bürgi, A., et al. 1995, Sol. Phys., 162, 441

Hsieh, K. C., Shih, K.L, Jokipii, J. R., \& Grzedzielski, S. 1992, ApJ, 393, 756

Hsieh, K. C., Giacalone, J., Czechowski, A., et al. 2010, ApJ, 718, L185

Izmodenov, V. V., \& Alexashov, D. B. 2006, in Physics of the inner heliosheath, 5th IGPP International Astrophysics Conference, ed. J. Heerikhuisen, V. Florinski, G. P. Zank, \& N. V. Pogorelov, AIP Conf. Proc., 858, 14

Kallenbach, R., Czechowski, A., Hilchenbach, M., \& Wurz, P. 2006, in The Physics of the Heliospheric Boundaries, ed. V. V. Izmodenov, \& R. Kallenbach, ISSI Scientific Report SR-005, Chap. 7, 203

Krimigis, S. M., Mitchell, D. G., Roelof, E. C., Hsieh, K. C., \& McComas, D. J. 2009, Science, 326, 971

Krimigis, S. M., Mitchell, D. G., Roelof, E. C., \& Decker, R. B. 2010, in Pickup ions throughout the heliosphere and beyond, Proc. 9th Annual Intl. Astrophysics Conference, Maui, Hawaii, 14-19 March, ed. J. A. le Roux, V. Florinsli, \& G. P. Zank, AIP Conf. Proc., 1302, 79

Krimigis, S. M., Roelof, E. C., Decker, R. B., \& Hill, M. E. 2011, Nature, 474, 359

le Roux, J. A., Potgieter, M. S., \& Ptuskin, V. S. 1996, J. Geophys. Res., 101, 4791

Lindsay, B. G., \& Stebbings, R. F. 2005, J. Geophys. Res., 110, A12213

McComas, D., Allegrini, F., Bochsler, P., et al. 2009, Science, 326, 959

Pogorelov, N. V., Zank, G. P., \& Ogino, T. 2008a, Adv. Space Res., 41, 306

Pogorelov, N. V., Heerikhuisen, J., \& Zank, G. P. 2008b, ApJ, 675, L41

Pryor, W., Gangopadhyay, P., Sandel, B., et al. 2008, A\&A, 491, 21

Ratkiewicz, R., \& Webb, G. M. 2002, J. Geophys. Res., 107, A12, SSH 11-1

Ratkiewicz, R., Barnes, A., Molvik, G. A., et al. 1998, A\&A, 335, 363

Richardson, J. D., Kasper, J. C., Wang, C., Belcher, J. W., \& Lazarus, A. J. 2008a, Nature, 454, 63

Richardson, J. D., Liu, Y., Wang, C., \& McComas, D. J. 2008b, A\&A, 491, 1

Scherer, K., \& Fahr, H.-J. 2003a, Geophys. Res. Lett., 30, 17-1

Scherer, K., \& Fahr, H.-J. 2003b, A\&A, 404, L47

Scherer, K., \& Ferreira, S. E. S. 2005, Astra, 1, 17

Schwadron, N. A., Bzowski, M., Crew, G. B., et al. 2009, Science, 326, 966

Schwadron, N. A., Allegrini, F., Bzowski, M., et al. 2011, ApJ, 731, 56

Sternal, O., Fichtner, H., \& Scherer, K. 2008, A\&A, 477, 365

Stone, E. C., Cummings, A. C., McDonald, F. B., et al. 2005, Science, 309, 2017, 2005

Stone, E. C., Cummings, A. C., McDonald, F. B., et al. 2007, in Proc. 30th International Cosmic Ray Conference, Merida, Mexico, ed. R. Caballero, J. C. D’Olivo, G. Medina-Tanco, L. Nellen, F. A. Sanchez, J. F. ValdesGalicia, Universidad Nacional Autonoma de Mexico, Mexico City, Mexico 2008, Vol. 1 (SH), 831

Stone, E. C., Cummings, A. C., McDonald, F. B., et al. 2008, Nature, 454, 71

Suess, S. T. 1990, Rev. Geophys., 28, 97

Webber, W. R., \& Intriligator, D. S. 2011, J. Geophys. Res., 116, A6

Zank, G. P. 1999, Space Sci. Rev., 89, 413

Zank, G. P., Pauls, H. L., Williams, L. L., \& Hall, D. T. 1996, J. Geophys. Res., 101,21639 